\title{
Subcritical water extraction of bioactive compounds from plants and algae: applications in pharmaceutical and food ingredients
}

\begin{abstract}
Plants and algae are the main sources of natural bioactive compounds used in the food and pharmaceutical industries. It is very important to achieve an efficient and safe technique to recover bioactive compounds while maintaining their quality and properties. Subcritical water extraction is the most promising engineering approach that offers an environmentally friendly technique for extracting various compounds from plants and algae. Application of pressurized water and high temperature in subcritical phase is able to modify the dielectric constant and polarity of the solvent which then contributes to a better extraction process. The technique improves the mass transfer rate and preserves the biological potency of the extracts. This article reviews current studies on the extraction of bioactive compounds from various species of plants and algae using the subcritical water technique and discusses its effects and benefits for the food and pharmaceutical industries.
\end{abstract}

Keyword: Algae; Bioactive compound; Plants; Subcritical water 